\title{
Estimation of the Exponential Distribution based on Multiply Progressive Type II Censored Sample
}

\author{
Kyeongjun Lee ${ }^{a}$, Chankeun Park ${ }^{b}$, Youngseuk Cho ${ }^{1, a}$ \\ ${ }^{a}$ Department of Statistics, Pusan National University \\ ${ }^{b}$ Department of Data Information, Korea Maritime University
}

\begin{abstract}
The maximum likelihood(ML) estimation of the scale parameters of an exponential distribution based on progressive Type II censored samples is given. The sample is multiply censored (some middle observations being censored); however, the ML method does not admit explicit solutions. In this paper, we propose multiply progressive Type II censoring. This paper presents the statistical inference on the scale parameter for the exponential distribution when samples are multiply progressive Type II censoring. The scale parameter is estimated by approximate ML methods that use two different Taylor series expansion types $\left(\mathrm{AMLE}_{I}, \mathrm{AMLE}_{I I}\right)$. We also obtain the maximum likelihood estimator(MLE) of the scale parameter $\sigma$ under the proposed multiply progressive Type II censored samples. We compare the estimators in the sense of the mean square error(MSE). The simulation procedure is repeated 10,000 times for the sample size $n=20$ and 40 and various censored schemes. The $\mathrm{AMLE}_{I I}$ is better than MLE and $\mathrm{AMLE}_{I}$ in the sense of the MSE.

Keywords: Approximate maximum likelihood estimator, exponential distribution, multiply progressive Type II censored sample, progressive Type II censored sample.
\end{abstract}

\section{Introduction}

Consider an exponential distribution with probability density function(pdf)

$$
g_{X}(x ; \sigma)=\frac{1}{\sigma} e^{-\frac{x}{\sigma}}, \quad x \geq 0, \sigma>0
$$

and cumulative density function(cdf)

$$
G_{X}(x ; \sigma)=1-e^{-\frac{x}{\sigma}}, \quad x \geq 0, \sigma>0 .
$$

The estimation of the parameters of the exponential distribution based on censored samples has been investigated by many authors such as Balakrishnan and Sandu (1996), Kang and Cho (1998), Kang and Park (2005), Singh and Kumar (2007), Asgharzadeh (2009), Chen and Lio (2010), and Shin et al. (2010). Balakrishnan and Sandu (1996) derived the best linear unbiased estimators(BLUEs) and maximum likelihood estimation under a general progressive Type II censored sample. Kang and Cho (1998) obtained the MRE for exponential distribution under general progressive Type II censored samples. Kang and Park (2005) derived an approximate maximum likelihood estimator(AMLE) for

\footnotetext{
This study was supported by the Research Fund Program of Research Institute for Basic Sciences, Pusan National University, Korea, 2011, Project No. RIBS-PNU-2011 301.

${ }^{1}$ Corresponding author: Associate Professor, Department of Statistics, Pusan National University, Busan 609-735, Korea.

E-mail: choys@pusan.ac.kr
} 


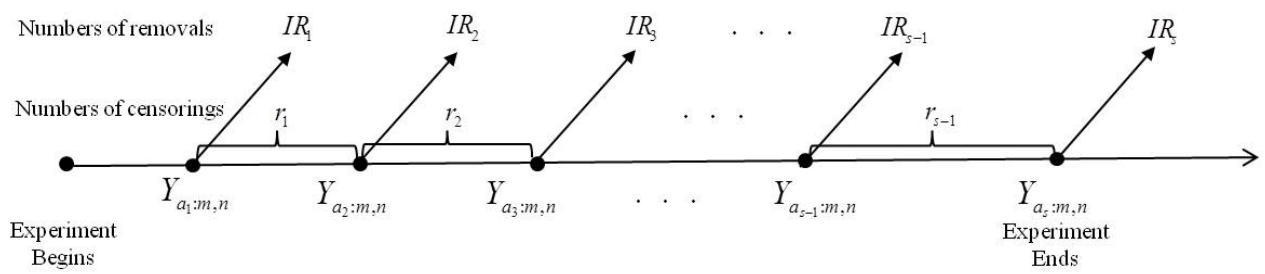

Figure 1: The multiply progressive Type II censoring schemes

the exponentiated exponential distribution based on a multiply Type II censored samples. Singh and Kumar (2007) derived a Bayes estimation based on a multiply Type II censoring scheme. Asgharzadeh (2009) derived an AMLE for the scaled generalized exponential distribution under progressive Type II censoring. Chen and Lio (2010) considered the parameter estimation for generalized exponential distribution under progressive Type I interval censoring. Shin et al. (2010) considered the parameter estimation for exponential distribution under progressive Type I interval censoring.

Many situations in life testing experiments exist where units are lost or removed from experimentation before failure. The loss may occur carelessly or unconsciously. For example, carelessly loss may occur in the case of accidental breakage of an experimental unit. Herd (1956) introduced a progressive censored sample. Under progressive censored sample, however, some units can be failed between two points of observation with exact times of failure of these units unobserved. For example, loss may arise in life-testing experiments when the failure times of some units were not observed due to mechanical or experimental difficulties. Therefore, the new censoring method is needed.

This study has two aims. The first is to propose multiply progressive Type II censoring. The second aim is to consider the MLEs of the scale parameter $\sigma$ when the data are multiply progressive Type II censored data. However, MLEs cannot be obtained in a closed form. We use the Bisection method and AMLEs to compute the MLEs. The rest of the paper is organized as follows. In Section 2, we propose a multiply progressive Type II censoring scheme. In Section 3, we derive some AMLEs of the $\sigma$ for the exponential distribution under the proposed multiply progressive Type II censoring samples. The $\sigma$ is estimated by an AMLE method using two different Taylor series expansion types.

\section{Multiply Progressive Type II Censoring}

Consider an experiment in which $n$ exponential components are put to test simultaneously and the failure times of these components are recorded, where $m$ is the preset number of failures to be observed. At the time of the first failure, $I R_{1}$ of the $n-1$ surviving units are randomly withdrawn from the life testing experiment. At the time of the next failures, $I R_{2}$ of the $n-2-I R_{1}$ surviving units are randomly and progressively withdrawn from the life testing experiment. Finally, at the time of the $m$ th failures, all the remaining $I R_{m}=n-m-I R_{1}-\cdots-I R_{m-1}$ surviving units are censored.

Let

$$
Y_{1: m: n} \leq Y_{2: m: n} \leq \cdots \leq Y_{m: m: n}
$$

be the resulting progressive Type II censored sample being the progressive censoring scheme. Then it is simple to derive the MLE of $\sigma$ (Balakrishnan and Sandu, 1996) as

$$
\hat{\sigma}=\frac{1}{m} \sum_{i=1}^{m}\left(I R_{i}+1\right) Y_{i: m: n} .
$$


Under the progressive Type II censoring scheme, suppose the experimenter fails to observe the middle $r$ observations. Now, let

$$
X_{a_{1}: m: n} \leq X_{a_{2}: m: n} \leq \cdots \leq X_{a_{s-1}: m: n} \leq X_{a_{s}: m: n}, \quad r_{i}=a_{i+1}-a_{i}-1, i=1,2, \ldots, s-1,
$$

be the available multiply progressive Type II censored sample. A schematic representation of the multiply progressive Type II censoring scheme is presented in Figure 1. The likelihood function based on (2.3) is given by

$$
L \propto \prod_{i=1}^{s} g\left(x_{a_{i}: m: n}\right)\left[1-G\left(x_{a_{i}: m: n}\right)\right]^{I R_{i}} \prod_{i=2}^{s}\left[G\left(x_{a_{i}: m: n}\right)-G\left(x_{a_{i-1}: m: n}\right)\right]^{r_{i-1}} .
$$

Let $Z_{a_{i}: m: n}=X_{a_{i}: m: n} / \sigma$ and then the variables $Z_{a_{i}: m: n}$ have a standard exponential distribution with

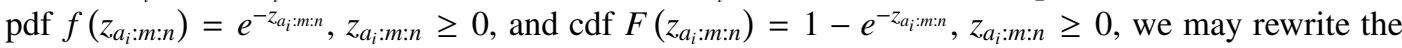
above likelihood function as

$$
L \propto \sigma^{-s} \prod_{i=1}^{s} f\left(z_{a_{i}: m: n}\right)\left[1-F\left(z_{a_{i}: m: n}\right)\right]^{I R_{i}} \prod_{i=2}^{s}\left[F\left(z_{a_{i}: m: n}\right)-F\left(z_{a_{i-1}: m: n}\right)\right]^{r_{i-1}} .
$$

Upon substituting for the functions $F(\cdot)$ and $f(\cdot)$, we obtain from (2.4) that

$$
\ln L \propto-s \ln \sigma+\sum_{i=1}^{s}\left\{\ln f\left(z_{a_{i}: m: n}\right)+I R_{i} \ln \left[1-F\left(z_{a_{i}: m: n}\right)\right]\right\}+\sum_{i=2}^{s} r_{i-1} \ln \left[F\left(z_{a_{i}: m: n}\right)-F\left(z_{a_{i-1}: m: n}\right)\right] .
$$

On differentiating the log-likelihood functions with respect to $\sigma$ of (2.5) and equation to zero, we obtain the estimating equations as

$$
\frac{\partial \ln L}{\partial \sigma}=-\frac{1}{\sigma}\left[s+\sum_{i=1}^{s}\left\{\left(1+I R_{i}\right) z_{a_{i}: m: n}\right\}+\sum_{i=2}^{s} r_{i-1}\left\{\frac{f\left(z_{a_{i}: m: n}\right) z_{a_{i}: m: n}-f\left(z_{a_{i-1}: m: n}\right) z_{a_{i-1}: m: n}}{F\left(z_{a_{i}: m: n}\right)-F\left(z_{a_{i-1}: m: n}\right)}\right\}\right]=0 .
$$

We can find the MLE $\hat{\sigma}$ of $\sigma$ that maximize the log-likelihood function in (2.5) by solving the (2.6). Some numerical methods must be employed since the (2.6) cannot be solved explicitly; therefore, we use the bisection method to obtain the numerical solution of the (2.6).

\section{Approximate Maximum Likelihood Estimators}

Since the (2.6) cannot be solved explicitly, it will be desirable to consider an approximation to the likelihood equations which provide us with explicit estimators for $\sigma$ (Kang, 2003).

Let

$$
\xi_{a_{i}: m: n}=F^{-1}\left(p_{a_{i}: m: n}\right)=-\ln \left(1-p_{a_{i}: m: n}\right)=-\ln \left(q_{a_{i}: m: n}\right),
$$

where

$$
p_{a_{i}: m: n}=1-\prod_{j=s-i+1}^{s} \frac{j+I R_{s-j+1}+\cdots+I R_{s}}{j+1+I R_{s-j+1}+\cdots+I R_{s}}, \quad i=1, \ldots, s .
$$

First, we expand the functions $f\left(z_{a_{i}: m: n}\right) /\left\{F\left(z_{a_{i}: m: n}\right)-F\left(z_{a_{i-1}: m: n}\right)\right\}$ and $f\left(z_{a_{i-1}: m: n}\right) /\left\{F\left(z_{a_{i}: m: n}\right)-\right.$ $\left.F\left(z_{a_{i-1}: m: n}\right)\right\}$ in Taylor series around the points $\xi_{a_{i}: m: n}$. 
Then, we can approximat the functions by

$$
\begin{aligned}
& \frac{f\left(z_{a_{i}: m: n}\right)}{F\left(z_{a_{i}: m: n}\right)-F\left(z_{a_{i-1}: m: n}\right)} \simeq \alpha_{1 i}+\beta_{1 i} z_{a_{i}: m: n}+\gamma_{1 i} z_{a_{i-1}: m: n}, \\
& \frac{f\left(z_{a_{i-1}: m: n}\right)}{F\left(z_{a_{i}: m: n}\right)-F\left(z_{a_{i-1}: m: n}\right)} \simeq \alpha_{2 i}+\beta_{2 i} z_{a_{i}: m: n}+\gamma_{2 i} z_{a_{i-1}: m: n},
\end{aligned}
$$

where

$$
\begin{aligned}
\alpha_{1 i} & =\frac{q_{a_{i}: m: n}}{p_{a_{i}: m: n}-p_{a_{i-1}: m: n}}\left[1+\xi_{a_{i}: m: n}+\frac{q_{a_{i}: m: n} \xi_{a_{i}: m: n}-q_{a_{i-1}: m: n} \xi_{a_{i-1}: m: n}}{p_{a_{i}: m: n}-p_{a_{i-1}: m: n}}\right], \\
\beta_{1 i} & =-\frac{q_{a_{i}: m: n}}{p_{a_{i}: m: n}-p_{a_{i-1}: m: n}}\left[1+\frac{q_{a_{i}: m: n}}{p_{a_{i}: m: n}-p_{a_{i-1}: m: n}}\right], \\
\gamma_{1 i} & =\frac{q_{a_{i}: m: n} q_{a_{i-1}: m: n}}{\left(p_{a_{i}: m: n}-p_{a_{i-1}: m: n}\right)^{2}}, \\
\alpha_{2 i} & =\frac{q_{a_{i-1}: m: n}}{p_{a_{i}: m: n}-p_{a_{i-1}: m: n}}\left[1+\xi_{a_{i-1}: m: n}+\frac{q_{a_{i}: m: n} \xi_{a_{i}: m: n}-q_{a_{i-1}: m: n} \xi_{a_{i-1}: m: n}}{p_{a_{i}: m: n}-p_{a_{i-1}: m: n}}\right], \\
\beta_{2 i} & =-\frac{q_{a_{i}: m: n} q_{a_{i-1}: m: n}}{\left(p_{a_{i}: m: n}-p_{a_{i-1}: m: n}\right)^{2}}, \\
\gamma_{2 i} & =-\frac{q_{a_{i-1}: m: n}}{p_{a_{i}: m: n}-p_{a_{i-1}: m: n}}\left[1-\frac{q_{a_{i-1}: m: n}}{p_{a_{i}: m: n}-p_{a_{i-1}: m: n}}\right] .
\end{aligned}
$$

By substituting the equations (3.1) and (3.2) into the equation (2.6), we may approximate the equation in (2.6) by

$$
\begin{aligned}
\frac{\partial \ln L}{\partial \sigma} \simeq & -\frac{1}{\sigma}\left[s-\sum_{i=1}^{s}\left(1+I R_{i}\right) z_{a_{i: m: n}}+\sum_{i=2}^{s} r_{i-1}\left\{\left(\alpha_{1 i}+\beta_{1 i} z_{a_{i: m: n}}+\gamma_{1 i} z_{a_{i-1: m: n}}\right) z_{a_{i: m: n}}\right.\right. \\
& \left.\left.-\left(\alpha_{2 i}+\beta_{2 i} z_{a_{i: m: n}}+\gamma_{2 i} z_{a_{i-1: m: n}}\right) z_{a_{i-1: m: n}}\right\}\right]=0 .
\end{aligned}
$$

The equation (3.3) is a quadratic equation in $\sigma$, with its roots given by

$$
\hat{\sigma}_{I}=\frac{B_{I}+\sqrt{B_{I}^{2}-4 s C_{I}}}{2 s},
$$

where

$$
\begin{aligned}
& B_{I}=\sum_{i=1}^{s}\left(1+I R_{i}\right) x_{a_{i}: m: n}-\sum_{i=2}^{s} r_{i-1}\left(\alpha_{1 i} x_{a_{i}: m: n}-\alpha_{2 i} x_{a_{i-1: m: n}}\right), \\
& C_{I}=\sum_{i=2}^{s} r_{i-1}\left(\beta_{1 i} x_{a_{i}: m: n}^{2}+2 \gamma_{1 i} x_{a_{i}: m: m} x_{a_{i-1: m: n}}-\gamma_{2 i} x_{a_{i-1: m: n}}^{2}\right) .
\end{aligned}
$$

Since $\beta_{1 i} x_{a_{i}: m: n}^{2}+2 \gamma_{1 i} x_{a_{i}: m: m} x_{a_{i-1: m: n}}-\gamma_{2 i} x_{a_{i-1: m: n}}^{2} \leq 0$, only one root is admissible.

Second, we expand the functions $\left\{f\left(z_{a_{i}: m: n}\right) z_{a_{i}: m: n}-f\left(z_{a_{i-1}: m: n}\right) z_{a_{i-1}: m: n}\right\} /\left\{F\left(z_{a_{i}: m: n}\right)-F\left(z_{a_{i-1}: m: n}\right)\right\}$ in Taylor series around the points $\xi_{a_{i}: m: n}$. 
Then, we can approximate the functions by the equations,

$$
\frac{f\left(z_{a_{i}: m: n}\right) z_{a_{i}: m: n}-f\left(z_{z_{i-1}: m: n}\right) z_{a_{i-1}: m: n}}{F\left(z_{a_{i}: m: n}\right)-F\left(z_{a_{i-1}: m: n}\right)} \simeq \alpha_{3 i}+\beta_{3 i} z_{a_{i}: m: n}+\gamma_{3 i} z_{a_{i-1}: m: n}
$$

where

$$
\begin{aligned}
\alpha_{3 i} & =\frac{q_{a_{i}: m: n} \xi_{a_{i}: m: n}^{2}-q_{a_{i-1}: m: n} \xi_{a_{i-1}: m: n}^{2}}{p_{a_{i}: m: n}-p_{a_{i-1}: m: n}}+\left[\frac{q_{a_{i}: m: n} \xi_{a_{i}: m: n}-q_{a_{i-1}: m: n} \xi_{a_{i-1}: m: n}}{p_{a_{i}: m: n}-p_{a_{i-1}: m: n}}\right]^{2}, \\
\beta_{3 i} & =\frac{q_{a_{i}: m: n}}{p_{a_{i}: m: n}-p_{a_{i-1}: m: n}}\left[1-\xi_{a_{i}: m: n}-\frac{q_{a_{i}: m: n} \xi_{a_{i}: m: n}-q_{a_{i-1}: m: n} \xi_{a_{i-1}: m: n}}{p_{a_{i}: m: n}-p_{a_{i-1}: m: n}}\right], \\
\gamma_{3 i} & =-\frac{q_{a_{i-1}: m: n}}{p_{a_{i}: m: n}-p_{a_{i-1}: m: n}}\left[1-\xi_{a_{i-1}: m: n}-\frac{q_{a_{i}: m: n} \xi_{a_{i}: m: n}-q_{a_{i-1}: m: n} \xi_{a_{i-1}: m: n}}{p_{a_{i}: m: n}-p_{a_{i-1}: m: n}}\right] .
\end{aligned}
$$

By substituting the equations (3.5) into the equation (2.6), we may approximate the equations in (2.6) by

$$
\frac{\partial \ln L}{\partial \sigma} \simeq-\frac{1}{\sigma}\left[s-\sum_{i=1}^{s}\left(1+I R_{i}\right) z_{a_{i}: m: n}+\sum_{i=2}^{s} r_{i-1}\left(\alpha_{3 i}+\beta_{3 i} z_{a_{i}: m: n}+\gamma_{3 i} z_{a_{i-1}: m: n}\right)\right]=0
$$

We can derive AMLE as follows;

$$
\hat{\sigma}_{I I}=\frac{B_{I I}}{C_{I I}}
$$

where

$$
\begin{aligned}
& B_{I I}=\sum_{i=1}^{s}\left(1+I R_{i}\right) x_{a_{i}: m: n}-\sum_{i=2}^{s} r_{i-1}\left(\beta_{3 i} x_{a_{i}: m: n}+\gamma_{3 i} x_{a_{i-1}: m: n}\right), \\
& C_{I I}=s+\sum_{i=2}^{s} r_{i-1} \alpha_{3 i} .
\end{aligned}
$$

\section{Illustrative Example and Simulated Results}

In this section, we present examples to illustrate the methods and assess the performance of estimators discussed in the previous sections.

\subsection{Real data}

Nelson (1982) gives data on times to the breakdown of an insulating fluid in an accelerated test conducted at various test voltages. The data are as follows:

$\begin{array}{rrrrrrrrrr}0.19 & 0.78 & 0.96 & 1.31 & 2.78 & 3.16 & 4.15 & 4.67 & 4.85 & 6.50 \\ 7.35 & 8.01 & 8.27 & 12.06 & 31.75 & 32.52 & 33.91 & 36.71 & 72.89 & \end{array}$

We apply the Kolmogorov Smirnov test to examine whether the data follow an exponential distribution. We obtained the $\operatorname{MLE}(\hat{\sigma}=14.3582)$ of the $\sigma$. Using this result, we create the critical value for the Kolmogorov test statistic for an exponential distribution by the Monte Carlo method. Since 
Table 1: Multiply progressive Type II censored data

\begin{tabular}{ccccccccccccc}
\hline \hline$a_{i}$ & 1 & 2 & 3 & 4 & 5 & 6 & 7 & 10 & 11 & 12 & 13 \\
\hline$x_{a_{i}: m: n}$ & 0.19 & 0.78 & 0.96 & 1.31 & 2.78 & 4.15 & 4.85 & 31.75 & 32.52 & 33.91 & 36.71 \\
$I R_{i}$ & 0 & 0 & 0 & 0 & 1 & 1 & 3 & 0 & 0 & 0 & 1 \\
$r_{i}$ & 0 & 0 & 0 & 0 & 0 & 0 & 2 & 0 & 0 & 0 & 0 \\
\hline \hline
\end{tabular}

Table 2: The relative mean squared errors and biases for the estimators of $\sigma$

\begin{tabular}{|c|c|c|c|c|c|c|}
\hline \multicolumn{7}{|c|}{$n=20$} \\
\hline \multirow[b]{2}{*}{$m$} & \multirow{2}{*}{$I R_{i}$} & \multirow[b]{2}{*}{$r$} & \multirow{2}{*}{$a_{i}$} & \multicolumn{3}{|c|}{ MSE(Bias) } \\
\hline & & & & MLE & $\mathrm{AMLE}_{I}$ & $\operatorname{AMLE}_{I I}$ \\
\hline 20 & $(20 * 0)$ & 0 & $1 \sim 20$ & $0.0507(0.0057)$ & $0.0507(0.0057)$ & $0.0507(0.0057)$ \\
\hline \multirow{11}{*}{18} & \multirow{6}{*}{$(17 * 0,2)$} & 0 & $1 \sim 18$ & $0.0568(0.0092)$ & $0.0568(0.0092)$ & $0.0568(0.0092)$ \\
\hline & & \multirow[b]{2}{*}{2} & $1 \sim 9,12 \sim 18$ & $0.0642(0.0069)$ & $0.0629(0.0384)$ & $0.0562(0.0019)$ \\
\hline & & & $1 \sim 4, \quad 7 \sim 18$ & $0.0641(0.0064)$ & $0.0618(0.0358)$ & $0.0568(0.0093)$ \\
\hline & & \multirow{3}{*}{4} & $1 \sim 3, \quad 8 \sim 18$ & $0.0643(0.0058)$ & $0.0651(0.0409)$ & $0.0570(0.0090)$ \\
\hline & & & $1 \sim 9,14 \sim 18$ & $0.0647(0.0076)$ & $0.0644(0.0424)$ & $0.0575(0.0113)$ \\
\hline & & & $1 \sim 4,7 \sim 9,12 \sim 18$ & $0.0643(0.0069)$ & $0.0694(0.0648)$ & $0.0562(0.0020)$ \\
\hline & \multirow{5}{*}{$(7 * 0,1,4 * 0,1,5 * 0)$} & 0 & $1 \sim 18$ & $0.0568(0.0092)$ & $0.0568(0.0092)$ & $0.0568(0.0092)$ \\
\hline & & \multirow{2}{*}{2} & $1 \sim 9,12 \sim 18$ & $0.0643(0.0070)$ & $0.0630(0.0387)$ & $0.0571(0.0098)$ \\
\hline & & & $1 \sim 4, \quad 7 \sim 18$ & $0.0641(0.0064)$ & $0.0618(0.0358)$ & $0.0568(0.0093)$ \\
\hline & & \multirow{2}{*}{4} & $1 \sim 3, \quad 8 \sim 18$ & $0.0643(0.0058)$ & $0.0651(0.0409)$ & $0.0570(0.0090)$ \\
\hline & & & $1 \sim 4,7 \sim 9,12 \sim 18$ & $0.0643(0.0070)$ & $0.0696(0.0652)$ & $0.0570(0.0102)$ \\
\hline \multirow{11}{*}{15} & \multirow{6}{*}{$(14 * 0,5)$} & 0 & $1 \sim 15$ & $0.0643(0.0073)$ & $0.0643(0.0073)$ & $0.0643(0.0073)$ \\
\hline & & \multirow{2}{*}{2} & $1 \sim 9,12 \sim 15$ & $0.0974(0.0052)$ & $0.0721(0.0406)$ & $0.0644(0.0080)$ \\
\hline & & & $1 \sim 4, \quad 7 \sim 15$ & $0.0974(0.0055)$ & $0.0721(0.0416)$ & $0.0644(0.0074)$ \\
\hline & & \multirow{3}{*}{4} & $1 \sim 3, \quad 8 \sim 15$ & $0.0975(0.0060)$ & $0.0740(0.0430)$ & $0.0645(0.0072)$ \\
\hline & & & $1 \sim 9,14 \sim 15$ & $0.1014(0.0076)$ & $0.0730(0.0397)$ & $0.0647(0.0077)$ \\
\hline & & & $1 \sim 4,7 \sim 9,12 \sim 15$ & $0.0975(0.0051)$ & $0.0850(0.0753)$ & $0.0645(0.0076)$ \\
\hline & \multirow{5}{*}{$(3 * 0,2,4 * 0,3,6 * 0)$} & 0 & $1 \sim 15$ & $0.0643(0.0073)$ & $0.0643(0.0073)$ & $0.0643(0.0073)$ \\
\hline & & \multirow{2}{*}{2} & $1 \sim 9,12 \sim 15$ & $0.0976(0.0047)$ & $0.0759(0.0474)$ & $0.0649(0.0088)$ \\
\hline & & & $1 \sim 4, \quad 7 \sim 15$ & $0.0937(0.0040)$ & $0.0731(0.0401)$ & $0.0644(0.0075)$ \\
\hline & & \multirow{2}{*}{4} & $1 \sim 4, \quad 9 \sim 15$ & $0.1016(0.0071)$ & $0.0740(0.0426)$ & $0.0650(0.0078)$ \\
\hline & & & $1 \sim 4,7 \sim 11,14 \sim 15$ & $0.0986(0.0066)$ & $0.0865(0.0802)$ & $0.0651(0.0090)$ \\
\hline
\end{tabular}

the critical value $(=0.3004)$ at the significance level of $\alpha=0.05$ exceed the corresponding values of the test statistics $(=0.2464)$, we can conclude that the data follow an exponential distribution. In this example, we have $n=19, m=13$, and $r=2$. The observations and censoring scheme are given in Table 1. From the (2.6), (3.4), and (3.7) the MLEs $\hat{\sigma}=18.3325$, the AMLEs $\hat{\sigma}_{I}=18.9059$, and $\hat{\sigma}_{I I}=18.5522$ are obtained.

\subsection{Simulation results}

To compare the performance of the proposed estimators of $\sigma$, we simulated the MSE and bias of all proposed estimators, by employing the Monte Carlo simulation method. The multiply progressive Type II censored data for sample size $n=20$ and 40, and various censoring schemes from the standard exponential distribution are generated using the algorithm presented in Balakrishnan and Sandhu (1995). Using this data, the MSEs of all proposed estimators are simulated by the Monte Carlo method based on 10,000 runs for the sample size $n=20$ and 40, and various choices of censoring under the multiply progressive Type II censored samples with $\sigma=1$. The simulation results are presented in Table 2 and Table 3, respectively. For simplicity in notation, we will denote the schemes $(0,0,0, \ldots, n-m)$ by $(m * 0, n-m)$. For example, $(7 * 0,2)$ denotes the progressive censoring scheme 
Table 3: The relative mean squared errors and biases for the estimators of $\sigma$

\begin{tabular}{|c|c|c|c|c|c|c|}
\hline \multicolumn{7}{|c|}{$n=40$} \\
\hline \multirow{2}{*}{$m$} & \multirow{2}{*}{$I R_{i}$} & \multirow{2}{*}{$r$} & \multirow{2}{*}{$a_{i}$} & \multicolumn{3}{|c|}{ MSE(Bias) } \\
\hline & & & & MLE & $\mathrm{AMLE}_{I}$ & $\mathrm{AMLE}_{I I}$ \\
\hline 40 & $(40 * 0)$ & 0 & $1 \sim 40$ & $0.0256(0.0040)$ & $0.0256(0.0040)$ & $0.0256(0.0040)$ \\
\hline \multirow{11}{*}{36} & \multirow{6}{*}{$(35 * 0,4)$} & 0 & $1 \sim 36$ & $0.0285(0.0039)$ & $0.0285(0.0039)$ & $0.0285(0.0039)$ \\
\hline & & \multirow{2}{*}{4} & $1 \sim 13,18 \sim 36$ & $0.0285(0.0038)$ & $0.0307(0.0218)$ & $0.0286(0.0039)$ \\
\hline & & & $1 \sim 25,30 \sim 36$ & $0.0285(0.0043)$ & $0.0309(0.0229)$ & $0.0286(0.0050)$ \\
\hline & & \multirow{3}{*}{8} & $1 \sim 11,20 \sim 36$ & $0.0287(0.0042)$ & $0.0310(0.0217)$ & $0.0288(0.0046)$ \\
\hline & & & $1 \sim 25,34 \sim 36$ & $0.0285(0.0046)$ & $0.0315(0.0249)$ & $0.0293(0.0087)$ \\
\hline & & & $1 \sim 13,18 \sim 27,32 \sim 36$ & $0.0287(0.0044)$ & $0.0346(0.0421)$ & $0.0289(0.0057)$ \\
\hline & \multirow{5}{*}{$(17 * 0,2,9 * 0,2,8 * 0)$} & 0 & $1 \sim 36$ & $0.0285(0.0039)$ & $0.0285(0.0039)$ & $0.0285(0.0039)$ \\
\hline & & \multirow{2}{*}{4} & $1 \sim 12,17 \sim 36$ & $0.0285(0.0039)$ & $0.0303(0.0221)$ & $0.0285(0.0039)$ \\
\hline & & & $1 \sim 21,26 \sim 36$ & $0.0287(0.0041)$ & $0.0307(0.0220)$ & $0.0287(0.0042)$ \\
\hline & & \multirow{2}{*}{8} & $1 \sim 8, \quad 17 \sim 36$ & $0.0285(0.0041)$ & $0.0307(0.0230)$ & $0.0285(0.0043)$ \\
\hline & & & $1 \sim 13,18 \sim 27,32 \sim 36$ & $0.0287(0.0040)$ & $0.0332(0.0403)$ & $0.0287(0.0042)$ \\
\hline \multirow{11}{*}{30} & \multirow{6}{*}{$(29 * 0,10)$} & 0 & $1 \sim 30$ & $0.0327(0.0069)$ & $0.0327(0.0069)$ & $0.0327(0.0069)$ \\
\hline & & \multirow{2}{*}{4} & $1 \sim 8, \quad 13 \sim 30$ & $0.0327(0.0070)$ & $0.0353(0.0266)$ & $0.0327(0.0070)$ \\
\hline & & & $1 \sim 20,25 \sim 30$ & $0.0329(0.0065)$ & $0.0359(0.0263)$ & $0.0327(0.0071)$ \\
\hline & & & $1 \sim 6, \quad 15 \sim 30$ & $0.0331(0.0073)$ & $0.0361(0.0284)$ & $0.0329(0.0067)$ \\
\hline & & 8 & $1 \sim 17,26 \sim 30$ & $0.0327(0.0070)$ & $0.0363(0.0280)$ & $0.0332(0.0077)$ \\
\hline & & & $1 \sim 8,13 \sim 20,25 \sim 30$ & $0.0327(0.0069)$ & $0.0396(0.0480)$ & $0.0327(0.0072)$ \\
\hline & \multirow{5}{*}{$(7 * 0,5,14 * 0,5,7 * 0)$} & 0 & $1 \sim 30$ & $0.0328(0.0070)$ & $0.0327(0.0069)$ & $0.0327(0.0069)$ \\
\hline & & \multirow{2}{*}{4} & $1 \sim 2, \quad 7 \sim 30$ & $0.0328(0.0070)$ & $0.0356(0.0285)$ & $0.0328(0.0070)$ \\
\hline & & & $1 \sim 15,20 \sim 30$ & $0.0329(0.0070)$ & $0.0357(0.0277)$ & $0.0329(0.0072)$ \\
\hline & & \multirow{2}{*}{8} & $1 \sim 9, \quad 18 \sim 30$ & $0.0329(0.0071)$ & $0.0365(0.0283)$ & $0.0330(0.0074)$ \\
\hline & & & $1 \sim 2,7 \sim 15,20 \sim 30$ & $0.0329(0.0071)$ & $0.0396(0.0493)$ & $0.0329(0.0073)$ \\
\hline
\end{tabular}

$(0,0,0,0,0,0,0,2)$.

From the Section 3, the MSEs of the estimators are simulated by the Monte Carlo method (based on 10,000 Monte Carlo runs) for sample size $n=20$ and 40, and various censoring schemes. We mainly compare the performances of the proposed estimators of $\sigma$, in terms of their biases and MSEs for various censoring schemes. From Table 2 and Table 3, the estimators $\hat{\sigma}_{I I}$ is generally more efficient than the $\hat{\sigma}_{I}$ and maximum likelihood estimator $\hat{\sigma}$.

From Table 2 and Table 3, the following general observations can be made. For all methods, the MSEs of all estimators decrease as sample size $n$ increases. For a fixed sample size, the MSE increases generally as the number of progressive censoring data $I R$ increases. For fixed sample and progressive censoring data size, the MSE increases generally as the number of missing data $r$ increases.

\section{Concluding Remarks}

In this paper, we propose the multiply progressive Type II censoring. We derive the AMLEs and the MLE of $\sigma$ in an exponential distribution based on a multiply progressive Type II censored sample. The parameter $\sigma$ is estimated by approximate maximum likelihood estimation method using two different types of Taylor series expansions. We evaluate the MSEs of the MLE of the $\sigma$ using the bisection method. We can see that $\hat{\sigma}_{I I}$ is generally more efficient than the $\hat{\sigma}_{I}$ and $\hat{\sigma}$.

In future studies, we will examine the estimation of the $\sigma$ in exponential distribution based on multiply progressive Type II censoring using a Bayesian method. We will also consider the estimation of the scale parameter $\sigma$ and location parameter $\mu$ in the exponential distribution based on multiply progressive Type II censoring. 


\section{References}

Asgharzadeh, A. (2009). Approximate MLE for the scaled generalized exponential distribution under progressive Type II censoring, Journal of the Korean Statistical Society, 38, 223-229.

Balakrishnan, N. and Sandu, R. A. (1995). A simple simulation algorithm for generating progressively Type II censored samples, The American Statistician, 49, 229-230.

Balakrishnan, N. and Sandu, R. A. (1996). Best linear unbiased and maximum likelihood estimation for exponential distributions under general progressive Type II censored sample, Sanky a: The Indian Journal of Statistics, 58, 1-9.

Chen, D. G. and Lio, Y. L. (2010). Parameter estimation for generalized exponential distribution under progressive Type I interval censoring, Computational Statistics and Data Analysis, 54, 1581-1591.

Herd, R. G. (1956). Estimation of the Parameters of a Population from a Multi-Censored Sample, Ph. D. Thesis, Iowa State College, Ames, Iowa.

Kang, S. B. (2003). Approximate MLEs for exponential distribution under multiply Type II censoring, Journal of the Korean Data and Information Science Society, 14, 983-988.

Kang, S. B. and Cho, Y. S. (1998). MRE for exponential distribution under general progressive Type II censored samples, Journal of the Korean Data and Information Science Society, 9, 71-76.

Kang, S. B. and Park, S. M. (2005). Estimation for the exponentiated exponential distribution based on multiply Type II censored samples, The Korean Communications in Statistics, 12, 643-652.

Nelson, W. (1982). Applied Life Data Analysis, John Wiley and Sons, New York.

Shin, H. J., Lee, K. H. and Cho, Y. S. (2010). Parameter estimation for exponential distribution under progressive Type I interval censoring, Journal of the Korean Data and Information Science Society, 21, 927-934.

Singh, U. and Kumar, A. (2007). Bayesian estimation of the exponential parameter under a multiply Type II censoring scheme, Austrian Journal of Statistics, 36, 227-238. 